\title{
Ferroelectric plasma source for heavy ion beam space charge neutralization
}

\author{
Philip C. Efthimion ${ }^{\mathrm{a}, *}$, Erik P. Gilson ${ }^{\mathrm{a}}$, Ronald C. Davidson ${ }^{\mathrm{a}}$, Larry Grisham ${ }^{\mathrm{a}}$, \\ B. Grant Logan ${ }^{b}$, Peter A. Seidl ${ }^{b}$, William Waldron ${ }^{b}$, Simon S. Yu ${ }^{b}$ \\ ${ }^{a}$ Plasma Physics Laboratory, Princeton University, Princeton, NJ 08543, USA \\ ${ }^{\mathrm{b}}$ Lawrence Berkeley National Laboratory, University of California, Berkeley, CA 94720, USA
}

Available online 1 March 2007

\begin{abstract}
Plasmas are a source of unbound electrons for charge neutralizing intense heavy ion beams to allow them to focus to a small spot size and compress their axial pulse length. The plasma source should be able to operate at low neutral pressures and without strong externally applied electric or magnetic fields. To produce $1 \mathrm{~m}$-long plasma columns, sources based upon ferroelectric ceramics with large dielectric coefficients are being developed. The sources utilize the ferroelectric ceramic $\mathrm{BaTiO}_{3}$ to form metal plasma. The drift tube inner surface of the Neutralized Drift Compression Experiment (NDCX) will be covered with ceramic material, and high voltage $(\sim 7 \mathrm{kV})$ will be applied between the drift tube and the front surface of the ceramics. A prototype ferroelectric source, $20 \mathrm{~cm}$ in length, has produced plasma densities of $5 \times 10^{11} \mathrm{~cm}^{-3}$. It was integrated into the Neutralized Transport Experiment (NTX), and successfully charge neutralized the $\mathrm{K}^{+}$ion beam. A $1 \mathrm{~m}$-long source comprised of five 20 -cm-long sources has been tested. Simply connecting the five sources in parallel to a single pulse forming network power supply yielded non-uniform performance due to the time-dependent nature of the load that each of the five plasma sources experiences. Other circuit combinations have been considered, including powering each source by its own supply. The 1-m-long source has now been successfully characterized, producing relatively uniform plasma over the $1 \mathrm{~m}$ length of the source in the mid- $10^{10} \mathrm{~cm}^{-3}$ density range. This source will be integrated into the NDCX device for charge neutralization and beam compression experiments.
\end{abstract}

(C) 2007 Elsevier B.V. All rights reserved.

Keywords: Plasma source; Neutralized transport; Beam neutralization

\section{Introduction}

Heavy ion fusion and high energy density physics applications utilize space-charge-dominated beams that need to be longitudinally and radially compressed to achieve the high beam intensities required at the target $[1,2]$. Longitudinal compression increases the beam current and reduces the size and cost of heavy ion drivers needed for these applications. Previously, the Neutralized Transport Experiment (NTX) demonstrated transverse focusing beyond the space-charge limit by space charge neutralizing the ion beam with background plasma [3]. Recently, the Neutralized Drift Compression Experiment (NDCX) demonstrated that an ion beam with axial velocity tilt

\footnotetext{
${ }^{*}$ Corresponding author. Tel.: + 16092433212.

E-mail address: pefthimion@pppl.gov (P.C. Efthimion).
}

could achieve longitudinal compression ratios of approximately 50 when the beam passed through a background plasma [4]. In this longitudinal compression experiment, plasma was carried out from a cathode arc source to a meter distance upstream using a solenoidal magnetic field. Here we present the development and characterization of a $1 \mathrm{~m}$-long plasma source to be incorporated in the longitudinal compression experiments on NDCX. This source is a linear scaling of the ferroelectric source that achieved charge neutralization and radial focusing of ion beams on the NTX device.

\section{Ferroelectric plasma source}

Ferroelectric materials have been intensively examined as high-current density electron emitters [5-7]. They have been projected to serve as large-surface area, high-current 
density cathodes. A mesh-like electrode structure is mounted to the emitting side of the ferroelectric material and the back surface has a metal plate electrode. A 3-15 kV potential is applied to the electrodes depending upon the thickness of the ferroelectric material. For ultra-thin-film ferroelectric materials, the applied voltage results in spontaneous polarization reversal on a nanosecond timescale, and a high electric field. Spontaneous polarization reversal yields a non-compensated charge at the surface and a high electron emission across the entire thin film.

For millimeter-thick ferroelectrics, the electric fields are too small to produce polarization reversal. However, plasma emission is observed and it is produced by electron emission from the vacuum micro-gaps between the dielectric surface and the edges of the metal electrode surface [8]. For this configuration, the value of the dielectric constant is the key factor. Commonly used ferroelectric materials have extremely large dielectric constants: $\mathrm{BaTiO}_{3}$ has a dielectric constant in the range of 1000-3000, and $\mathrm{Pb}(\mathrm{Zr}, \mathrm{Ti}) \mathrm{O}_{3}(\mathrm{PZT})$ has a dielectric constant in the range of 3000-6000. Once the threshold voltage is reached, plasma is formed over the entire surface of the dielectric. Typical current density yields are $0.5 \mathrm{~A} / \mathrm{cm}^{2}$. Typically, $8-16 \mathrm{kV}$, $0.25 \mu$ s pulses are applied to the electrodes at operating pressures near $10^{-5}$ Torr. Because the plasma is essentially all metal, the neutrals stick to the walls of the vacuum system and do not result in a significant pressure rise.

The characteristics of this plasma source are exactly what are required for the charge neutralization experiments on NTX and the beam compression experiments on NDCX. Furthermore, its ability to make the plasmaemitting layer arbitrarily long is important for the $1 \mathrm{~m}$-long plasma source. The source will be mounted on the walls of the drift tube just past the last focusing quadrupole magnet. The drift tube is approximately $7.5 \mathrm{~cm}$ in diameter. This small tube diameter will allow the density to be in the range of $10^{10}-10^{12} \mathrm{~cm}^{-3}$ on axis. The approach taken is to build a source with $4 \mathrm{~cm}$-long cylindrical ferroelectric pieces stacked together to form a $1 \mathrm{~m}$-long ferroelectric cylinder. Initially, a $20 \mathrm{~cm}$-long PZT ferroelectric source made of $2.54 \mathrm{~cm}$ long and $0.64 \mathrm{~cm}$ thick ferroelectric cylinders was built for evaluation (Fig. 1). The front surface electrode was made of $72,0.025 \mathrm{~cm}$ stainless steel wires strung along the length of the cylinder. The wires are mounted at each end of the source with an aluminium ring with 36 set screws. Each ring is mounted in a Delrin insulating sleeve to isolate it from the outer surface of the ferroelectric cylinders. The wires are pulled taught and actually hold the ferroelectric cylinders firmly together. Fig. 1 shows the wires mounted on one of the aluminium rings and the black Delrin insulating sleeve behind the rings. Also shown in the photograph is the copper wire that is wound around the outer surface of the ferroelectric cylinder to provide a good electrical contact. Electrically, the high-voltage pulse is applied to one of the aluminium mounting rings and the copper wire wound around the outer surface of the cylinder. All of the stainless steel wires

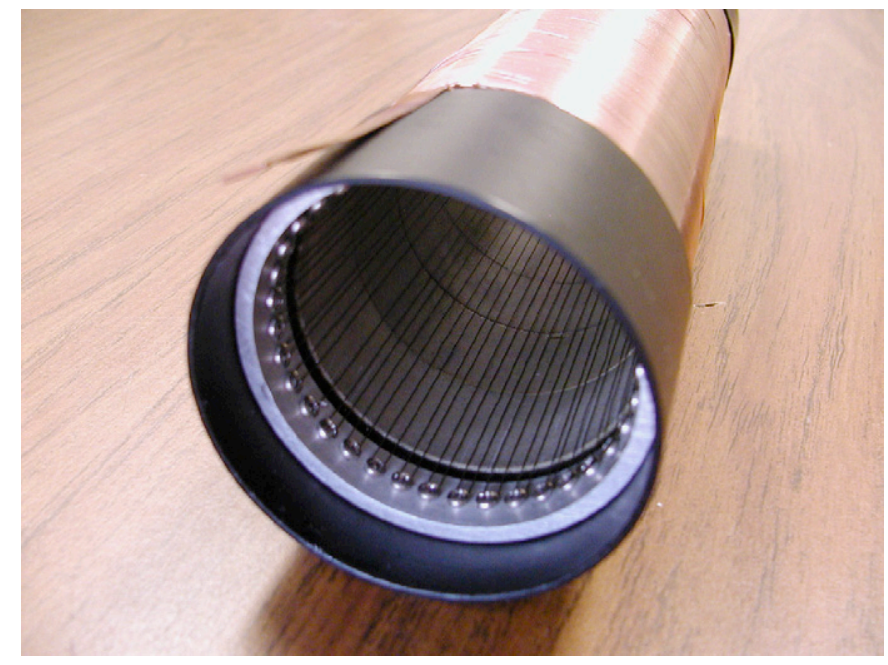

Fig. 1. Photograph of the $20 \mathrm{~cm}$-long PZT ferroelectric source.

are at the same potential through the aluminium rings at each end of the source.

The power supply for this pulsed source is a standard capacitor bank with a pulse-forming network to match the impedance of the source and maintain the $\mu$ s pulse shape. As presently configured, the pulse-forming network is matched to $4 \Omega$ and has a maximum output of $8 \mathrm{kV}$ and $2 \mathrm{kA}$. Thyratrons control the discharge of the charging capacitors. The output of the power supply is two $1 \mu \mathrm{s}$ pulses with an adjustable time delay between the pulses. For the $20 \mathrm{~cm}$-long PZT ferroelectric source, the typical operating parameters are $6 \mathrm{kV}$ and $800 \mathrm{~A}$. Langmuir probe measurements along the axis of the source show a somewhat constant density inside the cylindrical source. Since the source is pulsed, the electron density and temperature decays over $50 \mu$ s. Fig. 2 shows a profile of the electron density and temperature at times 10 and $25 \mu \mathrm{s}$ after the high-voltage pulse. The density is in the range of $10^{10}-10^{12} \mathrm{~cm}^{-3}$ at temperatures of $5-10 \mathrm{eV}$, respectively. With an ion beam density in NTX of $1 \times 10^{8} \mathrm{~cm}^{-3}$, the conditions in the PZT ferroelectric plasma source are sufficient to neutralize the ion beam space charge. Consequently, the plasma conditions can be controlled by adjusting either the applied high voltage or the delay when the ion beam passes through the plasma. With the ion beam pulse only $1 \mu \mathrm{s}$ long, it is easy to control the plasma conditions by adjusting the time when the beam pulse passes through the plasma.

The PZT ferroelectric source was then shipped to LBNL and incorporated in charge neutralization experiments on NTX. In the experiments, the last focusing quadrupole magnets direct the beam on a trajectory that focuses it to a spot size smaller than permitted by the space-charge limit. Before the beam approaches the space-charge limit, it enters the plasma created by the ferroelectric source, where it accumulates electrons and charge-neutralizes. The neutralized beam continues on its focusing trajectory, does not diverge, and achieves an extremely small spot size. 


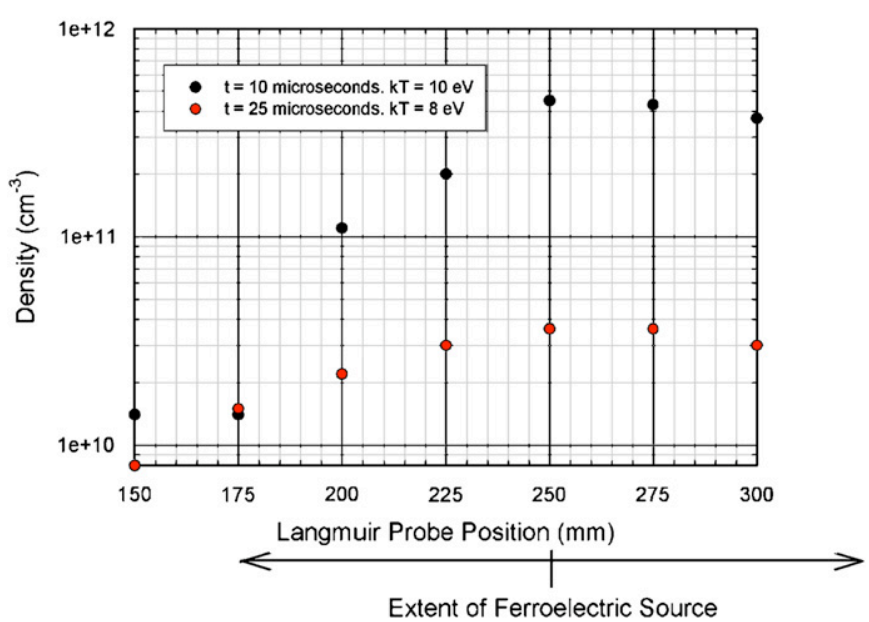

Fig. 2. Langmuir probe measurements along the axis of the PZT ferroelectric source.

Fig. 3 shows images of the beam cross-section before and after the neutralization. With the plasma source turned on, the beam achieves a spot size smaller than achievable without charge neutralization. The spot size was examined during a parameter scan of the plasma source voltage. The Langmuir probe measurements indicated that the plasma source current is proportional to the plasma electron density. In the scan, while the current increased the beam spot size decreased. Consequently, the scan showed that as the plasma density increased the spot size decreased, consistent with charge neutralization theory and simulations.

\section{Fabrication and characterization of the $1 \mathrm{~m}$-long ferroelectric plasma source}

The results from the PZT prototype ferroelectric source were sufficient to proceed with the design and building of the $1 \mathrm{~m}$-long plasma source for NDCX. It was fabricated with $\mathrm{BaTiO}_{3}$ ceramic instead of the PZT ceramic because the PZT ceramic ablated into a fine powder, and there was a concern for the hazard of the lead-based powder. A prototype 20-cm-long source did not work well with the thin wire electrodes used in the PZT, source and they were replaced by a stainless steel mesh. The mesh worked particularly well in producing plasma. The $1 \mathrm{~m}$-long source was fabricated as five $20-\mathrm{cm}$ sources separated by Delrin insulating rings with 1 in holes to provide diagnostic ports along the plasma. Fig. 4 shows a photograph of the entire source assembled, including the diagnostic ports. During the fabrication and testing, a number of rings were damaged and the source length was reduced from $1 \mathrm{~m}$ to $85 \mathrm{~cm}$.

Simply connecting the five sources in parallel to a single pulse-forming network power supply yielded non-uniform performance due to the time-dependent nature of the load that each of the five plasma sources experienced. Furthermore, the first two sources fabricated were extensively

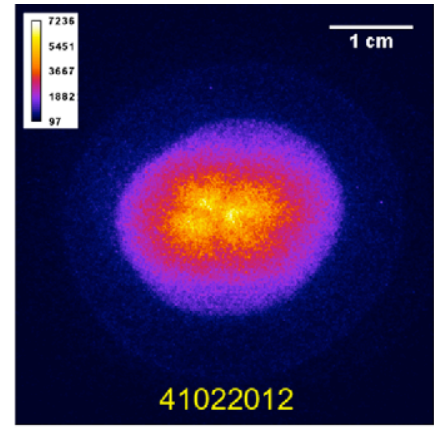

Before Neutralization

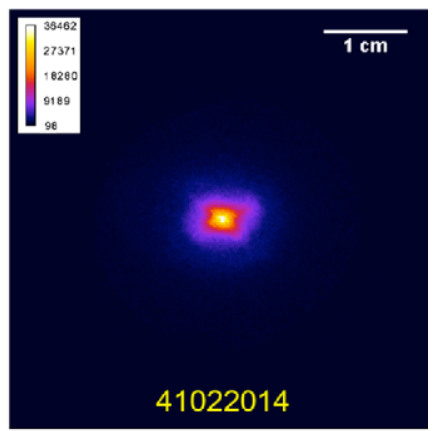

After Neutralization
Fig. 3. Images of the transverse beam spot size in NTX before and after neutralization using the PZT ferroelectric source.

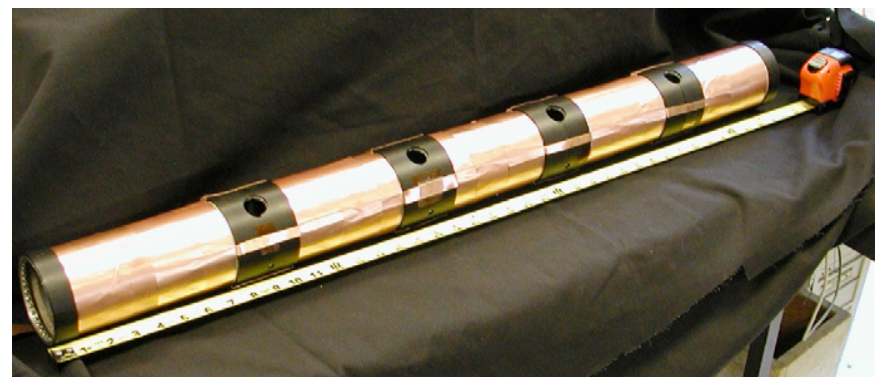

Fig. 4. The $1 \mathrm{~m}$-long $\mathrm{BaTiO}_{3}$ source consists of five individual sources with diagnostic ports between the sources.

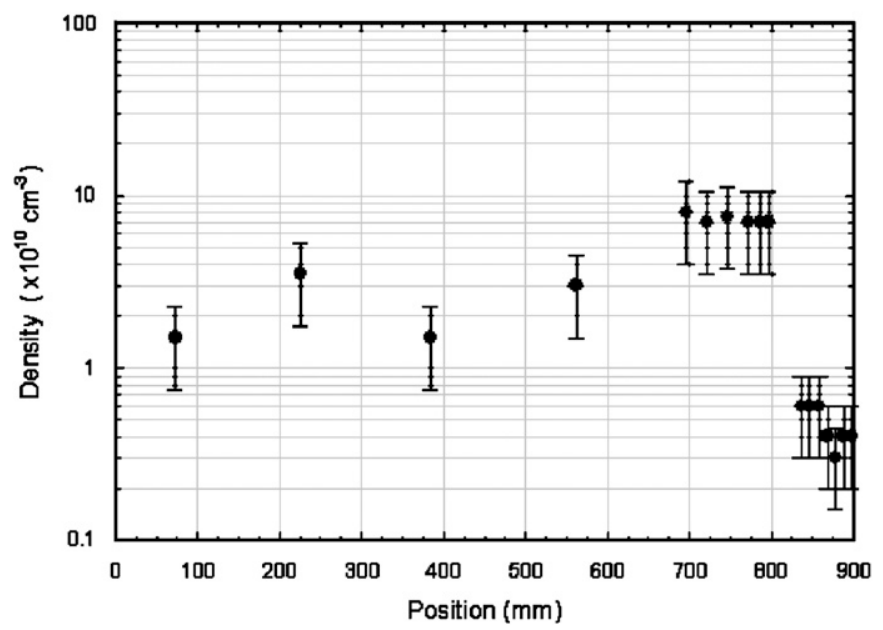

Fig. 5. Density measurements along the axis of the $\mathrm{BaTiO}_{3}$ plasma source indicate that the five sources are balanced in the mid $-10^{10} \mathrm{~cm}^{-3}$ density range.

tested compared to the remaining three. Consequently, the first two sources required higher voltage than the remaining three to achieve the same nominal density of mid$10^{10} \mathrm{~cm}^{-3}$. The power supply used for the earlier testing and described above provides two voltage pulses by two capacitor banks and pulse-forming networks charged with one voltage supply. With the variability in the five sources, the two banks and networks were electrically separated to independently charge the two groups of plasma sources. 
Each group of sources was connected in parallel across the outputs of each pulse-forming network. High-power resistors were added to the 3 newer sources to optimize the voltages applied to the sources to achieve densities in the mid $-10^{10} \mathrm{~cm}^{-3}$ range (Fig. 5). The resistors were necessary since each group of sources was supplied with the same voltage. The density along the entire source in Fig. 5 is reasonably uniform. Further resistor optimization can improve the uniformity if necessary. The lower density measurements in Fig. 5 located at $820-900 \mathrm{~mm}$ are near the plasma end or outside the source.

\section{Conclusions and future plans}

Characterization of the $\mathrm{BaTiO}_{3}$ plasma source is now complete, and the source has been shipped to LBNL for installation and use in the longitudinal compression experiments on NDCX. A velocity tilt is imposed on the beam pulse to compress the beam axially on NDCX, and, with this new plasma source, compression of the beam is expected to be as large as a factor of 50-100. Future plasma sources are being considered that are much longer and operate at higher densities to achieve very high compression values for high energy density physics applications.

\section{Acknowledgements}

This research was supported by the US Department of Energy under Contract No. DE-AC02-76-CHO-3073.

\section{References}

[1] B.G. Logan, D.A. Callahan, Nucl. Instr. and Meth. 415 (1998) 468.

[2] D.A. Callahan, Fusion Eng. Des. 441 (1996) 32-33.

[3] P.K. Roy, S. Yu, et al., Phys. Plasmas 11 (2004) 2890.

[4] P.K. Roy, et al., Phys. Rev. Lett 95 (2005) 234801.

[5] G. Rosenman, et al., Appl. Phys. Rev 88 (2000) 6109.

[6] A. Dunaevsky, et al., J. Appl. Phys. 95 (2004) 4621.

[7] A. Dunaevsky, et al., J. Appl. Phys. 90 (2001) 3689.

[8] A. Dunaevsky, et al., J. Appl. Phys. 89 (2001) 4480. 